\section{Brain, Behavior and Evolution}

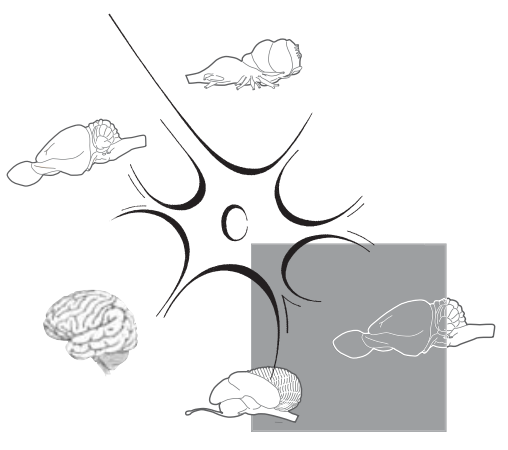

Scientific literature on the evolutionary origin of the neocortex is quite abundant and continued interest can be explained by our need to understand the origin of human cognitive abilities. In a recently published article, Dugas-Ford et al. [2012] analyze the origin of the neocortex using gene expression data at the cellular level, which they compare in representative mammals, birds and reptiles. They find molecular support for similarity between neurons in specific neocortical layers of mammals and specific neurons in the pallium of birds and reptiles [including neurons of the dorsal ventricular ridge (DVR)], thus reinforcing the cell-type homology hypothesis initially proposed by Karten [1969; 1997]. Any new contribution to our understanding of the organization and evolution of the pallium is welcome. However, in our opinion, the data of Dugas-Ford et al. [2012] are inconclusive for homology purposes, and the search for the evolutionary origin of the neocortex remains open. We explain our reasoning below, and add some thoughts on the contribution of evo-devo (evolutionary developmental biology) to understanding the mechanisms of evolution and the homology concept.
Published online: March 21, 2013

\title{
A Never-Ending Search for the Evolutionary Origin of the Neocortex: Rethinking the Homology Concept
}

\author{
Loreta Medina Antonio Abellán Ester Desfilis \\ Faculty of Medicine, Institute of Biomedical Research of Lleida (IRBLleida), University of \\ Lleida, Lleida, Spain
}

\section{Genes Chosen for Analyzing Homology}

Dugas-Ford and colleagues studied a number of genes that encode proteins related to various functional features of the cells (such as different ion channels or proteins related to cell homeostasis) or late development and functional maturation. However, not all genes play an equal role in morphological evolution and, therefore, not all genes are equally useful for identifying homologies. Current views on evolution, which incorporate evo-devo ideas, point to the relevance of developmental regulatory genes for explaining morphological evolution [Carroll, 2008]. In contrast to structural and house-keeping genes, the genes that regulate early development often encode proteins (transcription factors or morphogens) that play a crucial role in the formation and patterning of specific body structures. The function of these proteins is so important that their coding DNA sequences are under considerable evolutionary constraint and are, therefore, highly conserved across phyla (key functions of these regulatory proteins are often also conserved; see Gehring [2005]). Since their effects are dosage-dependent, there is also a constraint against duplication of these genes [Carroll, 2008]. On the other hand, the regulation of their expression is more complex than it is for structural/housekeeping genes. Whereas structural/housekeeping genes usually contain only one cisregulatory element (CRE), developmental regulatory genes contain multiple CREs, each driving the expression of the gene in a particular temporal and spatial sequence [Carroll, 2008]. Evolutionary variations in the CREs of developmental regulatory genes and/or variations in the activators or repressors binding the CREs, or in their cofactors, appear to have been a driving force for morphological evolution [Carroll, 2008]. Therefore, focus on the expression of developmental regulatory genes and on the regulation of their expression should provide us with better clues to understanding cortical evolution (or evolution of any other part of the brain or the body) and for the search of homologies.

\section{Cells and Areas Compared}

Dugas-Ford and colleagues analyzed the expression of a battery of genes in cells of different layers in the neocortex of mam-

\section{KARGER}

E-Mail karger@karger.com

www.karger.com/bbe
2013 S. Karger AG, Base

0006-8977/13/0813-0150\$38.00/0
Loreta Medina

Universitat de Lleida, Institut de Recerca Biomèdica de Lleida (IRBLleida) Department Medicina Experimental - Edif. Biomedicina I, Lab. Brain Development and Evolution, ES-25198 Lleida (Spain)

E-Mail loreta.medina@mex.udl.cat 
mals (mouse and ferret), and compared them to cells showing similar expression profiles in the dorsal cortex of a reptile (the red-eared turtle) as well as to the hyperpallium and DVR of two avian species (chicken and zebra finch). The genes Eag2/Kcnh5 (a potassium ion channel) and Rorb/Nr1f2 (retinoid-related orphan receptor-beta; a transcription factor gene related to circadian rhythms) were used as neocortical layer 4 markers. In the avian and reptilian pallium, they were found in thalamorecipient areas, including the entopallium and L field of avian DVR, the interstitial nucleus of the avian apical hyperpallium (HA) and the turtle dorsal cortex. Six genes were used as markers of neocortical layer 5: the transcription factor genes Er81/Etv1 (which targets genes that modulate cell proliferation and differentiation in different tissues and is involved in maturation and synaptic function of cerebellar circuitry [Abe et al., 2011]) and Fezf2/Znf312 (which controls the development of dendrites in layer 5 pyramidal neurons, and is possibly involved in fasciculation of their projections), the ion channel gene Cacna1h, the sulfatase gene Sulf2, the gene Pcp4 (encoding a calmodulin-binding protein that promotes neuronal or neuroendocrine cell differentiation and neurotransmitter release $[\mathrm{Ha}$ rashima et al., 2011]) and one gene of uncertain function, Tmem200a/Kiaa1913. In the avian and reptilian pallium, most of these genes were expressed in neurons with long descending projections, including neurons in the avian arcopallium and HA, and the reptilian dorsal cortex.

Although the authors use these data to defend homology between layer-specific neocortical neurons and specific neuron groups in the avian and reptilian pallium, some of the genes employed in this study are not specific to particular neocortical layers, as they are also expressed in other parts of the pallium, including the hippocampal formation (Eag2, Rorb, ER81, Fezf2, Cacna1h, Sulf2, Pcp4 and Tmem200a), the pallial amygdala (Eag2, Rorb, ER81, Fezf2, Cacna1h and Pcp4) and/or the olfactory bulb (Eag2, Rorb, Er81, Cacna1h, Sulf2 and Pcp4) (see also [Saganich et al., 2001] and the Allen Brain Atlas). Therefore, these genes are only general markers of thalamorecipient pallial neurons (including both lemnothalamorecipient and collothalamorecipient neurons; [Butler, 1994a, b]), or descending projection pallial neurons (assuming that they can be shown to have these specific features in all pallial areas).
In addition, it is worth noting that some of the studied genes are also expressed in parts of the subpallium (and in other parts of the brain; see the Allen Brain Atlas) and can therefore not be used to distinguish pallial from subpallial neurons, either individually or in combination. This is likely due to the fact that the genes employed by Dugas-Ford et al. [2012] are not involved in early developmental events, but are rather related to the late maturation and/or functional activity of the neurons.

\section{Homology of Genes, Cell Types or Brain Regions: Are They Complementary?}

Karten's original proposal of homology between layer-specific neocortical neurons and specific neuron groups in the avian and reptilian pallium was based on the similarity of connections. In contrast, Dugas-Ford et al. [2012] use molecular data to draw conclusions about such cell-type homology. However, as noted above, the presented molecular data support only a more general homology between thalamorecipient pallial cells or between descending projection pallial neurons of different amniotes.

How useful are claims about cell-type homologies, and how do they relate to the homology of gene networks and brain regions? To deal with such questions, some comparative neurobiologists have invoked the hierarchical nature of biological organization, proposing that genes, developmental processes, morphological structures, physiological function and behaviors all constitute different levels of organization and that homology at any of the levels does not necessarily imply homology at the other levels [Striedter and Northcutt, 1991; Striedter, 1999]. In this sense, homology at the cellular level can be useful as a tool to identify comparable cell types in different species, such as thalamorecipient cells of the pallium. If proven that the genetic networks involved in the production of these cells are the same, it could be a case of deep homology as proposed by Shubin et al. [2009] for photoreceptors, which would not necessarily imply homology of the pallial subdivisions in which those cells are found.

However, the nervous system of vertebrates contains millions or billions of neurons (depending on the species) and each neuron is unique, as unique is its specific set of connections. In such a system, it is ex- tremely difficult to define specific neuron types if we do not refer to regions or fields. For example, it is useful to distinguish the motor neurons born in the midbrain (oculomotor) from those in rhombomere 2 (trigeminal) or those in specific cervical spinal segments (as noted below, the specific embryonic origin within the neural tube will determine the distinct muscles each motor neuron group innervates). Similarly, in the pallium, it would be useful to distinguish between thalamorecipient cells in the neocortex and those found in other parts of the pallium (e.g. the amygdala). Moreover, it would make little sense to compare neocortical neurons to cerebellar cortical neurons or to thalamic neurons, even if they express similar sets of genes (e.g. ER81 and Fezf2 are expressed in neocortical layer 5 and in the cerebellar cortex; Allen Brain Atlas). Therefore, to get meaningful definitions of specific neuron types, such as pallial neurons versus subpallial neurons or subtypes within these major groups, we find it necessary to refer to the location of these neurons in the nervous system.

The most useful approach for defining the basic divisions of the nervous system and comparing them across species is to analyze the expression patterns of multiple genes involved in embryonic brain development within the context of a topological structural framework [Puelles et al., 2000; Puelles and Medina, 2002; Puelles and Ferran, 2012]. The combination of regulatory proteins expressed in each progenitor zone controls the features of its adult derivatives, including neurotransmitter(s), neuropeptide(s), membrane receptors, ion channels and adhesion molecules as well as connections. Therefore, a cell's embryonic origin determines much of its adult identity [Puelles and Medina, 2002; Medina, 2007].

In the case of the vertebrate pallium, at least four subdivisions have been observed in the embryonic brain of different amniotes: medial, dorsal, lateral and ventral pallia [Puelles et al., 2000; Abellán et al., 2009; Medina and Abellán, 2009; Medina et al., 2011]. These embryonic pallial subdivisions have similar topological positions and express similar combinations of developmental regulatory genes. Based on these features, these pallial subdivisions have been proposed to be field-homologous across amniotes. For example, the embryonic medial pallium is located dorsomedially and expresses the transcription factors Emx1, Lhx2, Lhx9, Pax6 and Tbr1 in mouse, chicken and some reptiles, such as 
turtles or lizards [Fernandez et al., 1998; Puelles et al., 2000; Abellán et al., 2009; Desfilis et al., 2012]. The embryonic dorsal pallium in mouse and chicken and its putative corresponding area in reptiles is located in a dorsal position within the pallium, and is characterized by expression of the transcription factors Emx1, Lhx2, Pax6 and Tbr1, but a lack of Lhx9 [Fernandez et al., 1998; Puelles et al., 2000; Abellán et al., 2009; Desfilis et al., 2012]. In contrast, the lateroventral pallium (lateral plus ventral subdivisions) of mouse, chicken and reptiles is located more laterally, abuts the boundary with the striatum ventrally, contains the lateral olfactory tract at its surface [Striedter, 1997] and expresses Lhx2, Pax6, Tbr1, Lmo3 and Lhx9 [Puelles et al., 2000; Abellán et al., 2009; Desfilis et al., 2012] (data on Lmo3 only known in mouse and chicken). In particular, in different amniotes, the embryonic ventral pallium lacks ventricular expression of Emx1 [Fernandez et al., 1998; Puelles et al., 2000], but expresses Lhx9 in the ventricular or subventricular zone and in the mantle (later in development, Lhx9 expression becomes restricted to rostral, bulbar and caudal amygdalar levels of the ventral pallium) [Abellán et al., 2009; Desfilis et al., 2012]. In the mouse, the embryonic ventral pallium is additionally characterized by the expression of Dbx1 [Yun et al., 2001; Medina et al., 2004], but data on Dbx1 in nonmammals are incomplete and inconclusive [Bielle et al., 2005].

In the mouse, mutations in developmental regulatory genes that are expressed strongly in the dorsomedial pallium (such as Lhx2) have dramatic effects on the development of this pallial territory (and its derivatives, the hippocampal formation and neocortex), but the lateroventral pallium (and the piriform lobe that derives from it) remains fairly unchanged based on gene expression patterns (e.g. spared expression of Lmo3 [Vyas et al., 2003]). This type of evidence supports the notion that dorsomedial and lateroventral pallial divisions are truly different progenitor zones and produce different structures.

As the mammalian neocortex derives from the dorsal pallium, while the DVR of reptiles and birds derives from the lateroventral pallium, these data disfavor homology of the neocortex and DVR. On the contrary, such data would favor homology between the claustroamygdaloid region in mammals and the DVR in birds and reptiles, since both derive from the lateroven- tral pallium [Striedter, 1997; Puelles, 2001; Medina et al., 2011].

However, as noted by Striedter and Northcutt [1991] (also [Striedter 1999]), embryonic homology does not necessarily imply homology of all adult derivatives. So, the dorsal pallium produces the neocortex in mammals and the Wulst (hyperpallium) in birds [Striedter, 1997; Puelles, 2001], but can we safely say that the neocortex is homologous to the Wulst? Similarly, the lateroventral pallium produces the claustroamygdaloid region in mammals and the DVR in reptiles and birds, but does this imply one-to-one homology between their adult derivatives? Homologous embryonic domains have clearly undergone divergent evolution in different lineages. Some of their adult derivatives are easily homologized across species, whereas others are much less clear. For example, the mammalian neocortex, the avian Wulst and the reptilian dorsal cortex (which apparently includes a sector derived from the dorsal pallium) seem to contain a homologous primary visual area receiving input from the thalamic dorsal geniculate nucleus, which was likely present in the amniotes' common ancestor [Butler, 1994a, b; Medina and Reiner, 2000]. However, some cell types in this area, such as those involved in corticocortical (or palliopallial) connections, may have evolved independently in mammals and birds [Reiner, 1991]. A pending issue is that, for now, we lack a set of developmental regulatory genes that clearly distinguishes the embryonic dorsal pallium from the adjacent pallia (many of the regulatory genes that characterize the dorsal pallium are also expressed in the adjacent medial pallium and/or the lateral pallium [Medina and Abellán, 2009]). Therefore, a clear definition of a true dorsal pallium in reptiles is still missing, which is essential for deciphering the evolutionary origin of the neocortex.

Furthermore, tangential migrations of cells between domains during development can complicate the analysis of homology between adult structures of different animals. For example, although the mammalian neocortex originates from the dorsal pallium, it includes neuron subpopulations derived from the subpallium (i.e. the GABAergic interneurons [Marín and $\mathrm{Ru}-$ benstein, 2001]). This tangential migration of GABAergic interneurons from the subpallium to the pallium has been found in different vertebrates, even lampreys [Medina and Abellán, 2009]. In addition, the neocortex also contains a transient sub- population of glutamatergic cells derived from the ventral pallium, which dies postnatally [Puelles, 2011]. These cells include transient Cajal-Retzius cells, but also other cells that show a dispersed location across layers (i.e. they do not occupy any specific cortical layer); this has only been described in mammals [Puelles, 2011].

Another complication in the effort for analyzing the homology of the adult structures and their cellular components is that derivatives of different, nonhomologous embryonic domains may have acquired similar features, to perform similar functions, by convergent evolution. This may have involved convergence at the level of function-related genes. For example, the independent evolution of infrared vision in rattlesnakes, pythons and boas involves the convergent evolution of similar functionrelated genes [Yokoyama et al., 2011]. Therefore, the neurons and neuronal circuits involved in complex behaviors may well have evolved independently in different vertebrates. This may be the case for some neural circuits of the songbird DVR and the mammalian neocortex, both subserving some similar cognitive functions, such as vocal learning and communication [Jarvis et al., 2005] or perception of human faces [Marzluff et al., 2012]. It is important to state that nonhomology does not diminish, but, on the contrary, increases the interest in their study for trying to understand, for example, which are the neural requirements for a certain function. Moreover, it is marvellous to see and to try to understand how evolution can find independent solutions for a similar functional achievement.

In conclusion, the analysis of homologies at different levels (genetic, cellular or regional) is useful and complementary for trying to decipher evolution of brain complexity. However, for acquiring deeper conclusions on specific homologies of adult structures, or their cellular components, it is extremely important to refer to their embryonic origin and the genetic regulatory networks involved in their early development, as these are usually highly conserved in evolution and help to reveal the morphotypic organization of different brains. Moreover, it is also very important to identify the developmental changes that are responsible for evolutionary variation and divergence, or the developmental mechanisms responsible for evolutionary convergence. For both of these, more comparative data are necessary. 


\section{References}

- Abe H, Okazawa M, Nakanishi S (2011): The Etv1/Er81 transcription factor orchestrates activity-dependent gene regulation in the terminal maturation program of cerebellar granule cells. Proc Natl Acad Sci USA 108:1249712502 .

-Abellán A, Legaz I, Vernier B, Rétaux S, Medina L (2009): Olfactory and amygdalar structures of the chicken ventral pallium based on the combinatorial expression patterns of LIM and other developmental regulatory genes. J Comp Neurol 516:166-186.

Bielle F, Griveau A, Narboux-Nême N, Vigneau S, Sigrist M, Arber S, Wassef M, Pierani A (2005): Multiple origins of Cajal-Retzius cells at the borders of the developing pallium. Nat Neurosci 8:1002-1012.

Butler AB (1994a): The evolution of the dorsal pallium in the telencephalon of amniotes: cladistic analysis and a new hypothesis. Brain Res Brain Res Rev 19:66-101.

Butler AB (1994b): The evolution of the dorsal thalamus of jawed vertebrates, including mammals: cladistic analysis and a new hypothesis. Brain Res Brain Res Rev 19:29-65.

Carroll SB (2008): Evo-devo and an expanding evolutionary synthesis: a genetic theory of morphological evolution. Cell 134:25-36.

Desfilis E, Abellán A, Medina L (2012): Expression of developmental regulatory genes help to delineate the pallial amygdala in a lacertic lizard. Soc Neuroci Abstr 894:10.

Dugas-Ford J, Rowell JJ, Ragsdale CW (2012): Cell-type homologies and the origins of the neocortex. Proc Natl Acad Sci USA 109: 16974-16979.

Fernandez AS, Pieau C, Repérant J, Boncinelli E, Wassef M (1998): Expression of the Emx-1 and Dlx-1 homeobox genes define three molecularly distinct domains in the telencephalon of mouse, chick, turtle and frog embryos: implications for the evolution of telencephalic subdivisions in amniotes. Development 125:2099-2111.

Gehring WJ (2005): New perspectives on eye development and the evolution of eyes and photoreceptors. J Hered 96:171-184.

-Harashima S, Wang Y, Horiuchi T, Seino Y, Inagaki N (2011): Purkinje cell protein 4 positively regulates neurite outgrowth and neurotransmitter release. J Neurosci Res 89 1519-1530.
Karten HJ (1969): The organization of the avian telencephalon and some speculations on the phylogeny of the amniote telencephalon. Ann NY Acad Sci 167:164-179.

Karten HJ (1997): Evolutionary developmental biology meets the brain: the origins of mammalian cortex. Proc Natl Acad Sci USA 94: 2800-2804.

Jarvis ED, Güntürkün O, Bruce L, Csillag A, Karten H, Kuenzel W, Medina L, Paxinos G, Perkel DJ, Shimizu T, Striedter G, Wild JM, Ball GF, Dugas-Ford J, Durand SE, Hough GE, Husband S, Kubikova L, Lee DW, Mello CV, Powers A, Siang C, Smulders TV, Wada K, White SA, Yamamoto K, Yu J, Reiner A, Butler AB, Avian Brain Nomenclature Consortium (2005): Avian brains and a new understanding of vertebrate brain evolution. Nature Rev Neurosci 6:151-159.

Marín O, Rubenstein JLR (2001): A long, remarkable journey: tangential migration in the telencephalon. Nat Rev Neurosci 2:780-790.

Marzluff JM, Miyaoka R, Minoshima S, Cross DJ (2012): Brain imaging reveals neuronal circuitry underlying the crow's perception of human faces. Proc Natl Acad Sci USA 109: $15912-15917$

Medina L (2007): Field Homologies; in Kaas JH, Striedter GF and Rubenstein JLR (eds): Evolution of Nervous Systems: A Comprehensive Reference. Volume 1: Theories, Development, Invertebrates. Amsterdam, Elsevier Academic Press, pp 73-87.

Medina L, Abellán A (2009): Development and evolution of the pallium. Semin Cell Dev Biol 20:698-711.

Medina L, Bupesh M, Abellán A (2011): Contribution of genoarchitecture to understanding forebrain evolution and development, with particular emphasis on the amygdala. Brain Behav Evol 78:216-236.

Medina L, Legaz I, González G, de Castro F, Rubenstein JLR, Puelles L (2004): Expression of Dbx1, Neurogenin 2, Semaphorin 5A, Cadherin 8, and Emx1 distinguish ventral and lateral pallial histogenetic divisions in the developing claustroamygdaloid complex. J Comp Neurol 474:504-523.

Medina L, Reiner A (2000): Do birds possess homologues of mammalian primary visual, somatosensory and motor cortices? Trends Neurosci 23:1-12.

Puelles L (2001): Thoughts on the development, structure and evolution of the mammalian and avian telencephalic pallium. Philos Trans R Soc Lond B Biol Sci 356:1583-1598.
Puelles L (2011): Pallio-pallial tangential migrations and growth signaling: new scenario for cortical evolution? Brain Behav Evol 78:108127

Puelles L, Ferran JL (2012): Concept of neural genoarchitecture and its genomic fundament. Front Neuroanat 6:47.

Puelles L, Kuwana E, Puelles E, Bulfone A, Shimamura K, Keleher J, Smiga S, Rubenstein JLR (2000): Pallial and subpallial derivatives in the embryonic chick and mouse telencephalon, traced by the expression of the genes Dlx-2, Emx-1, Nkx-2.1, Pax-6, and Tbr-1. J Comp Neurol 424:409-438.

Puelles L, Medina L (2002): Field homology as a way to reconcile genetic and developmental variability with adult homology. Brain Res Bull 57:243-255.

Reiner A (1991): A comparison of neurotransmitter-specific and neuropeptide-specific neuronal cell types present in the dorsal cortex of reptiles with those present in the isocortex of mammals. Brain Behav Evol 38: 53-91.

Saganich MJ, Machado E, Rudy B (2001): Differential expression of genes encoding subthreshold-operating voltage-gated $\mathrm{K}+$ channels in brain. J Neurosci 21:4609-4624.

Shubin N, Tabin C, Carroll S (2009): Deep homology and the origins of evolutionary novelty. Nature 457:818-823.

Striedter GF (1997): The telencephalon of tetrapods in evolution. Brain Behav Evol 49:179213

Striedter GF (1999): Homology in the nervous system: of characters, embryology and levels of analysis. Novartis Found Symp 222:158170.

Striedter GF, Northcutt RG (1991): Biological hierarchies and the concept of homology. Brain Behav Evol 38:177-189.

Vyas A, Saha B, Lai E, Tole S (2003): Paleocortex is specified in mice in which dorsal telencephalic patterning is severely disrupted. J Comp Neurol 466:545-553.

Yokoyama S, Altun A, DeNardo DF (2011): Molecular convergence of infrared vision in snakes. Mol Biol Evol 28:45-48.

Yun K, Potter S, Rubenstein JLR (2001): Gsh2 and Pax6 play complementary roles in dorsoventral patterning of the mammalian telencephalon. Development 128:193-205. 